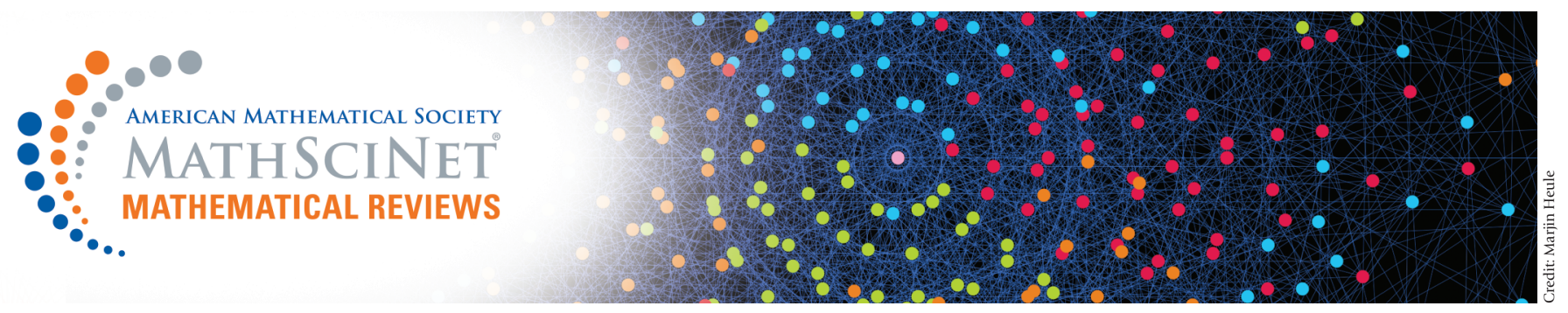

\title{
Mathematics Subject Classification 2020
}

\section{Edward Dunne and Klaus Hulek}

The latest revision of the Mathematics Subject Classification (MSC) has been published, replacing the 2010 Mathematics Subject Classification (referred to as MSC2010). Searchable versions are available from the zbMATH site (https:// zbmath.org/classification/) and the MathSciNet site (https://mathscinet.ams.org/mathscinet/searchMSC .html .

Mathematical Reviews (MR) and zbMATH collaborate on maintaining the Mathematics Subject Classification, which is used by these reviewing services, publishers, funding agencies, and others to categorize items in the mathematical sciences literature. It is a taxonomy created by and for mathematical researchers. Every ten years, the two editorial groups solicit input from the mathematics community. For the current revision, we received more than 350 comments and suggestions from more than 100

Edward Dunne is the executive editor of Mathematical Reviews. His email address is egd@ams.org.

Klaus Hulek is the editor-in-chief of zbMATH. His email address is hulek amath.uni-hannover.de.

This article will also appear in the March 2020 edition of the EMS Newsletter.

For permission to reprint this article, please contact: reprint-permission aams.org.

DOI: https://dx.doi.org/10.1090/noti2052 different people. MR and zbMATH carefully considered this input from the community and used it in the preparation of our joint revision of the classification.

As anticipated, there are no changes at the two-digit level, but several at the three-digit level, and hundreds at the five-digit level. Nine new three-digit classes were added: $18 \mathrm{M}$ Monoidal categories and operads, $18 \mathrm{~N}$ Higher categories and homotopical algebra, 53E Geometric evolution equations, 57K Low-dimensional topology in specific dimensions, 57Z Relations of manifolds and cell complexes with science and engineering, 60L Rough analysis, 62R Statistics on algebraic and topological structures, $68 \mathrm{~V}$ Computer science support for mathematical research and practice, and $82 \mathrm{M}$ Basic methods in statistical mechanics. For five-digit classes, 113 classes were retired and 486 new classes were introduced. The new MSC contains 63 twodigit classifications, 529 three-digit classifications, and 6,006 five-digit classifications.

There were some general changes. Descriptions of classes were changed to be more useful when searching online or via database interfaces. Previous descriptions assumed the user was looking at a full list of the classifications, which would provide context. An example of the limitation is a search of MSC2010 for "optimization," which returns 18 matches, not counting essentially every class in 49 Calculus of variations and optimal control; optimization. There 
were three classes named "Flow control and optimization": 76B75, 76D55, and 76N25. The three different contexts were incompressible inviscid fluids, incompressible viscous fluids, and compressible fluids and gas dynamics. Now, they have descriptions with more detail, as in $76 \mathrm{~B} 75$ Flow control and optimization for incompressible inviscid fluids. There were three classes just named "Optimization" in the areas 74P Mechanics of deformable solids, 78M50 Optics, electromagnetic theory, and 80M50 Classical thermodynamics, heat transfer. Now they have descriptions that include the context, as in 78M50 Optimization problems in optics and electromagnetic theory.

In previous versions of the MSC, there were some "hyphen classes" of the form XX-00 General reference works, XX-01 Introductory expositions, XX-02 Research exposition, XX-03 History, XX-04 Software, and XX-06 Proceedings, conferences, collections, etc., along with scattered other hyphen classes. The use of hyphen classes has been made more uniform across the MSC, so that most two-digit classes now have these five subclasses. Some hyphen classes would be redundant and are omitted, such as the nonexistent class 01-03, since the two-digit class for "History of mathematics and mathematicians" does not need a subclass for history. The classes $\mathbf{- 0 8}$ for Computational methods for problems from the parent class, $-\mathbf{1 0}$ for Mathematical modeling or simulation for problems from the parent class, and $\mathbf{- 1 1}$ for Research data for problems from the parent class have been added where appropriate. For example, there is now the class 20-08 Computational methods for problems from group theory, and the class 20-11 Research data for problems from group theory. An example of an omission of one of these new hyphen classes for reasons of redundancy is $\mathbf{6 5}$ Numerical analysis, which does not need the $\mathbf{- 0 8}$ class for computational methods. Also, some classes have alternatives to $\mathbf{- 0 8}$ with more detail, such as the eight five-digit classes in the three-digit class 14Q Computational aspects in algebraic geometry. The hyphen classes $\mathbf{- 1 0}$ mostly occur for applied classes, namely MSCs 70 through $\mathbf{9 4}$, as in $\mathbf{7 0 - 1 0}$ Mathematical modeling or simulation for problems from mechanics of particles and systems.

The influences of data and computation on the mathematical sciences are reflected in the classes. In addition to the -08 classes, and not including classes from 03 Mathematical logic and foundations or $\mathbf{6 8}$ Computer science, there are 58 classes referring to computations, computational methods, or computers. For instance, for MSC2020, two new classes, 14Q25 Computational algebraic geometry over arithmetic ground fields and 14Q30 Computational real algebraic geometry, have been added to the three-digit class 14Q Computational aspects in algebraic geometry, which had been added to the MSC in 1991. Similarly, two new classes were added under $37 \mathrm{M}$ Approximation methods and numerical treatment of dynamical systems: 37M21 Computational methods for invariant manifolds of dynamical systems and 37M22 Computational methods for attractors of dynamical systems. For the - 11 classes, examples of the types of data envisioned include statistical data, mathematical tables, collections of mathematical objects and their properties, such as integer sequences (as found in the OEIS, for instance), or databases of modular forms or Calabi-Yau varieties. In addition to the - $\mathbf{1 1}$ classes, there are 31 classes with specific instances of data, including the new classes 62R10 Functional data analysis, 62R40 Topological data analysis, and 68P27 Privacy of data.

Mathematical Reviews and zbMATH are now using MSC2020 as their classification schemes. We welcome and encourage the community also to adopt the MSC2020. It is available from msc2020. org in PDF or TeX. A SKOS version will be available later.

The classification is jointly published by the two organizations under a Creative Commons CC-BY-NC-SA license. Corrections to possible errors in the new system can be submitted by email to feedback@msc2020.org. All information about MSC2020 is jointly shared by MR and zbMATH.

The editors and staffs at Mathematical Reviews and zbMATH express their gratitude to the numerous members of the community for their assistance in this lengthy revision process.

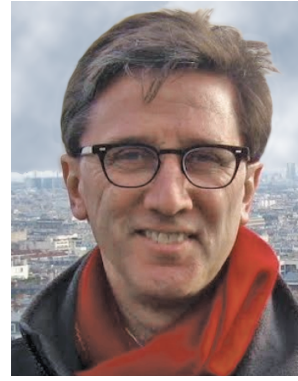

Edward Dunne



Klaus Hulek

\section{Credits}

Photo of Dunne is courtesy of Edward Dunne. Photo of Hulek is by Sebastian Gerhard. 